\title{
Magnetic activity in HD 111456, a young F5-6 main-sequence star ${ }^{\star}$
}

\author{
R. Freire Ferrero ${ }^{1}$, A. Frasca ${ }^{2}$, E. Marilli ${ }^{2}$, and S. Catalano ${ }^{2}$ \\ 1 Observatoire Astronomique, 11 rue de l'Université, 67000 Strasbourg, France \\ 2 INAF - Osservatorio Astrofisico di Catania, via S. Sofia 78, 95123 Catania, Italy
}

Received 21 November 2002 / Accepted 30 September 2003

\begin{abstract}
We present spectroscopic and photometric observations of the young F5-6 V field star HD 111456 which exhibits emission cores in the $\mathrm{Ca}$ II $\mathrm{H}$ and $\mathrm{K}$ lines and a filling in the $\mathrm{H} \alpha$ line. Absorption lines of $\mathrm{He} \mathrm{I}_{3}$ and Li I are clearly detected and we give a new value of $v \sin i=41.5 \mathrm{~km} \mathrm{~s}^{-1}$. Although the limit of magnetic activity detectability at optical wavelengths occurs around F5-6 spectral types, HD 111456 shows unusual chromospheric activity. High UV fluxes in the C II, C IV, Si II and Si IV lines and a strong X-ray emission confirm that HD 111456 is one of the most active F5-6 V star. The Ca II and $\mathrm{H} \alpha$ chromospheric emission fluxes do not show any detectable variation on time scales of few days, as well as $u v b y$ photometry. A possible explanation in terms of a very high level of magnetic activity and a homogeneous distribution of active regions, both at chromospheric and photospheric level, or a very low inclination of the rotation axis with respect to the line of sight is proposed. From 1998 to 2001 the radial velocity varies indicating a possible binary system, but the spectral distribution of HD 111456 is better reproduced by a single normal F5 V star, and composite spectra simulations (Ca II, H $\alpha$ ) with a normal inactive $\mathrm{F}$ star and a very active low-mass $\mathrm{K}-\mathrm{M}$ star are not able to reproduce the observed emissions.
\end{abstract}

Key words. stars: activity - stars: individual: HD 111456

\section{Introduction}

Within a wide program aimed to study the chromospheric and coronal activity in stars with a shallow convective zone we examine in detail the case of the young main sequence star HD 111456 (= HR 4867, F5-6 V, $V=5.85, B-V=0.46$ ). HD 111456 is a single star of the nucleus of the UMa moving group (300-400 Myr old), whose membership is confirmed both by kinematics and spectroscopic criteria mainly based on the high level of Ca II H \& K emission obtained from the Mt. Wilson spectrophotometric $S$ index (Eggen 1992; Soderblom \& Mayor 1993; Chupina et al. 2001).

Furthermore, the conspicuous activity of HD 111456 at chromospheric and TR level is stressed by the UV emission lines, like C II $\lambda 1335$, Si IV $\lambda 1400$ and C IV $\lambda 1550$, seen in lowresolution IUE spectra (Simon \& Landsman 1991; Soderblom $\&$ Clements 1987) and by the strong emission in the cores of the $\mathrm{h}$ and $\mathrm{k} \mathrm{Mg}$ II lines observed at high resolution by Vladilo et al. (1987). This behaviour clearly appears in Fig. 1, where the $\mathrm{C}$ IV versus the $\mathrm{C}$ II surface flux for a sample of main-sequence

Send offprint requests to: R. Freire Ferrero, e-mail: freire@cluster.u-strasbg.fr

* Based on observations collected at the Observatoire de Haute Provence (CNRS), France, and at the Osservatorio Astrofisico di Catania, Italy.

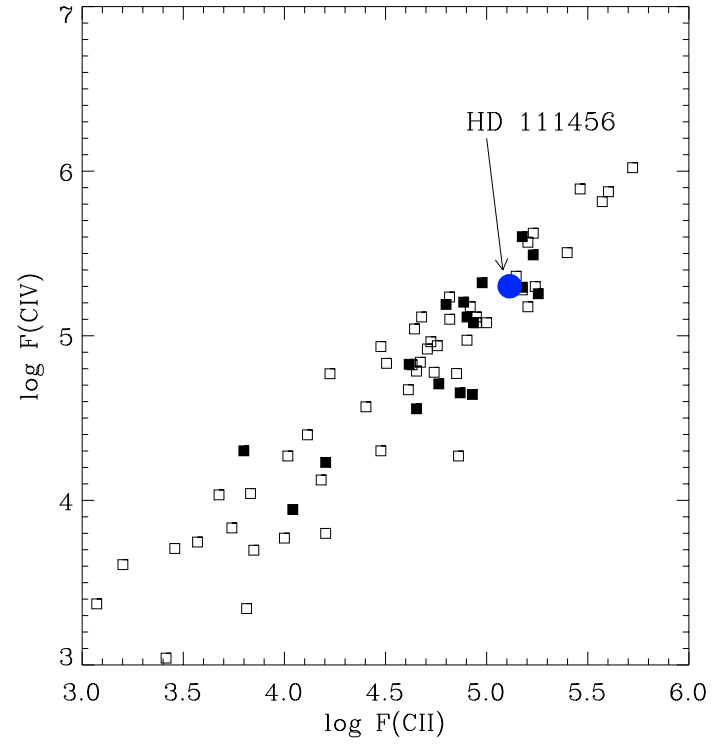

Fig. 1. Location of HD 111456 (big dot) into a flux-flux diagram for a sample of main-sequence stars (data from Rutten et al. 1991). The F-type stars are displayed with filled squares.

stars from Rutten et al. (1991) is shown. HD 111456 falls in the region of the most active F-type stars. 
HD 111456 displays also high values of X-ray emission (Schmitt et al. 1985; Hünsch et al. 1999) compared to those of field stars of similar spectral type.

Further evidence of the strong activity is given by the detection of the $\mathrm{HeI}_{3}$ line (Garcia Lopez et al. 1993), a clear indication that HD 111456 lies in the HR diagram well beyond the "activity boundary", i.e. the limit at which the magnetic activity starts and the associated rotational braking is efficient. In this case, the observed rotational speed $v \sin i$ of $39 \mathrm{~km} \mathrm{~s}^{-1}$ (Wolff \& Simon 1997) is appropriated for a young MS star which has not yet been effectively spun down by magnetic braking. Its young age is also supported by the detection of the Li I $\lambda 6708$ absorption line (Soderblom et al. 1993a), with a Li abundance just below the Pleiades values. Actually, it occupies the midF stars region for which a deep decrease in the lithium abundance, the so-called lithium chasm, is observed in Hyades stars (Boesgaard \& Tripicco 1986). In addition, active F-type stars display an unusual behaviour of chromospheric and TR emission lines. For example, the early-F and mid-F Hyades stars do not follow the general trend of increasing activity with more rapid rotation displayed by cooler stars (Bohm-Vitense et al. 2001, 2002).

Although Hyades stars of mid-F spectral type, in many cases, do show rotational modulation of $\mathrm{Ca}$ II $\mathrm{H}$ and $\mathrm{K}$ emission (Baliunas et al. 1985; Donahue et al. 1996), no clear modulation or cyclic variation have been detected for HD 111456 in the long-term survey performed by Baliunas et al. (1995).

No attempt to search for short- and long-term brightness changes connected to rotational modulation produced by surface inhomogeneities or to solar-type cycles has been made for HD 111456 so far. On the other hand a recent survey of Radick et al. (1998) on photometric pattern variations in field stars does not exclude a possible variability at the limit of detectability threshold of $\simeq 0.005 \mathrm{mag}$, more evident for long-term observations. Hence, young field stars, as HD 111456, are suitable candidates to present evidences of an observable photospheric activity.

In this work we study HD 111456 with the aim to better understand the behaviour of the photospheric and chromospheric activity in a young active mid-F single star. Here we present and discuss new spectroscopic high-resolution observations in the $\mathrm{H}$ and $\mathrm{K}$ lines of $\mathrm{Ca}$ II, $\mathrm{H} \alpha, \mathrm{H} \beta, \mathrm{He} \mathrm{I} \mathrm{D}_{3}$, and Li I lines.

\section{Observations and data reduction}

\subsection{Optical Strömgren photometry}

The photometric observations have been carried out with the $91-\mathrm{cm}$ Cassegrain telescope at the M.G. Fracastoro station (Serra La Nave, Mt. Etna) of the Osservatorio Astrofisico di Catania (OAC). These observations were performed with a photon-counting photometer equipped with an EMI 9893QA/350 photomultiplier, cooled to $-15^{\circ} \mathrm{C}$.

We observed HD 111456 during 9 nights from July 24th to August 5th 2000. HD 111270 and HD 111337 were used as comparison and check stars, respectively.

A set of $u v b y$ standard stars of known Strömgren indices (Lindemann \& Hauck 1973) was nightly observed to transform
Table 1. Log of the spectroscopic observations.

\begin{tabular}{|c|c|c|c|c|}
\hline Date & $\begin{array}{l}\mathrm{UT}_{\text {midexp }} \\
\mathrm{h} \mathrm{m}\end{array}$ & Instr. & $\begin{array}{c}R \\
\lambda / \Delta \lambda\end{array}$ & $\begin{array}{l}\text { Wav. range } \\
(\AA)\end{array}$ \\
\hline 1998 June 18 & 2115 & AURELIE & 22000 & $3880-4020$ \\
\hline “ “ 28 & 2118 & AURELIE & 22000 & $3880-4020$ \\
\hline 1998 July 02 & 2240 & ELODIE & 42000 & $3906-6818$ \\
\hline “" 03 & 2107 & ELODIE & 42000 & $3906-6818$ \\
\hline “ “ 05 & 2037 & ELODIE & 42000 & $3906-6818$ \\
\hline 2000 July 10 & 2344 & REOSC & 15000 & $5850-6690$ \\
\hline “ “ 11 & 2240 & REOSC & 15000 & $5850-6690$ \\
\hline “ “ 14 & 2117 & REOSC & 15000 & $5850-6690$ \\
\hline “ “ 15 & 2148 & REOSC & 15000 & $5850-6690$ \\
\hline “ “ 15 & 2255 & REOSC & 15000 & $5850-6690$ \\
\hline “ “ 17 & 2315 & REOSC & 15000 & $5850-6690$ \\
\hline 2001 July 12 & 2143 & REOSC & 15000 & $5850-6690$ \\
\hline
\end{tabular}

the instrumental magnitudes of HD 111456 into the Strömgren photometric system.

\subsection{Optical spectroscopy}

We obtained spectra with different resolutions for HD 111456 (Table 1) at the Observatoire de Haute Provence (OHP) and at the M.G. Fracastoro station (Serra La Nave, Mt. Etna) of the OAC, within a survey program of chromospheric activity on $\mathrm{F}$ and $\mathrm{G}$ stars belonging to clusters and moving groups of different ages.

The two spectra acquired with the AURELIE spectrograph at the 152-cm coudé telescope (OHP), using the grating N. 7 (Gillet et al. 1994), have been centred in the Ca II H and $\mathrm{K}$ region.

The échelle spectra obtained with the ELODIE spectrograph at the 193-cm telescope of the OHP (Baranne et al. 1996), allow us to study simultaneously the $\mathrm{Ca}$ II $\mathrm{H}$ and $\mathrm{K}$ lines, the $\mathrm{H} \beta$ and $\mathrm{H} \alpha$ lines, the $\mathrm{He} \mathrm{I}_{3} 5876 \AA$ line, the Li I $6708 \AA$ line, and a number of photospheric lines through all the optical spectrum.

The observations at the M.G. Fracastoro station of the OAC were performed with the REOSC échelle spectrograph connected to the 91-cm telescope through an optical fibre (UV - NIR, $200 \mu \mathrm{m}$ core diameter). A thinned back-illuminated SITe CCD of $1024 \times 1024$ pixels was used to record the spectra.

To produce reference absorption profiles not affected by activity signatures and to perform the spectral synthesis analysis (Frasca et al. 2000, hereafter Paper I), we selected inactive, slowly-rotating stars of similar spectral type as HD 111456, namely HD 216385 (F6 IV-V, $B-V=0.47, v \sin i=$ $5.9 \mathrm{~km} \mathrm{~s}^{-1}$ ), HD 181096 (F6 IV, $B-V=0.463, v \sin i=$ $3 \mathrm{~km} \mathrm{~s}^{-1}$ ) and Procyon A (F5 IV-V, $B-V=0.42, v \sin i=$ $3 \mathrm{~km} \mathrm{~s}^{-1}$ ). The low activity degree of these stars has been mainly inferred from UV chromospheric and TR lines as displayed by IUE spectra (see e.g. the Mg II lines in Fig. 7), so that we expect negligible or no emission in the $\mathrm{H} \alpha$ or $\mathrm{Ca}$ II $\mathrm{H}$ and K cores (Cayrel et al. 1983; Zarro \& Rodgers 1983; Herbig 1985). 
We observed Procyon at the OHP with ELODIE in January 24th, 1997, while the spectra of HD 181096 and HD 216385 have been kindly provided by the ELODIE database (OHP).

Several radial-velocity (RV) standard stars have also been observed both at the OHP and OAC in the same observing runs, with the aim to study possible RV variations.

The reduction of AURELIE spectra was performed by the ONEDESPEC task of IRAF ${ }^{1}$. Since we are dealing with the very deep $\mathrm{H}$ and $\mathrm{K}$ lines, particular attention was paid to the background subtraction that was performed using several offset exposures.

The ELODIE spectra were automatically reduced on-line during the observations. The echelle orders of ELODIE spectra have been normalized to the local continuum by a low-order polynomial fit.

The correction for scattered light for ELODIE and AURELIE spectra follows the same procedure as that described in Paper I.

The reduction of the OAC spectra was performed using the ECHELLE task of IRAF package, following the standard steps of background subtraction, division by a flat field spectrum given by a halogen lamp, wavelength calibration by means of the emission lines of a Thorium-Argon lamp, normalization through a polynomial fit to the continuum.

The red part of the spectra is contaminated by several water vapour and $\mathrm{O}_{2}$ telluric lines. We have removed these lines at the $\mathrm{H} \alpha$ and $\mathrm{Na}$ I $\mathrm{D}_{2}$ wavelengths using the spectra of $\alpha \mathrm{Cep}$ (A7 IV, $v \sin i=196 \mathrm{~km} \mathrm{~s}^{-1}$ ) and Altair (A7 V, $v \sin i=220 \mathrm{~km} \mathrm{~s}^{-1}$, Freire Ferrero et al. 1995; $v \sin i=217 \mathrm{~km} \mathrm{~s}^{-1}$, Royer et al. 2002), which have a very broad and shallow $\mathrm{H} \alpha$ profile and serve, after a proper normalization, as templates for the water vapour lines (see Paper I, Fig. 1).

\section{Data analysis and results}

\section{1. uvby photometry}

In Fig. 2 the individual differential magnitude measurements are displayed versus time, together with the comparison-check $(\mathrm{C}-\mathrm{Ck})$ ones. The standard deviation of all $\mathrm{C}-\mathrm{Ck}$ values is 0.011 both in $y$ and $b$ bands, while for the variable-comparison $(\mathrm{V}-\mathrm{C})$ we find 0.014 and 0.013 in $y$ and $b$ filters, respectively. Since all stars have a similar magnitude and the exposure times have been chosen to obtain at least 150000 counts in each filter, the effect of photon statistics is the same for $\mathrm{V}, \mathrm{C}$ and $\mathrm{Ck}$ and completely negligible with respect to errors due to sky instability, seeing variation, etc. The standard deviation of HD 111456 data is not significantly higher than that of C-Ck data so that a real variability of HD 111456 cannot be assessed only on the basis of this argument.

On the whole, the differential magnitudes in $y$ and $b$ Strömgren filters seem to show low amplitude $\left(\sim 0^{\mathrm{m}} 03\right)$

${ }^{1}$ IRAF is distributed by the National Optical Astronomy Observatory, which is operated by the Association of University for Research in Astronomy, inc. (AURA) under cooperative agreement with the National Science Foundation.
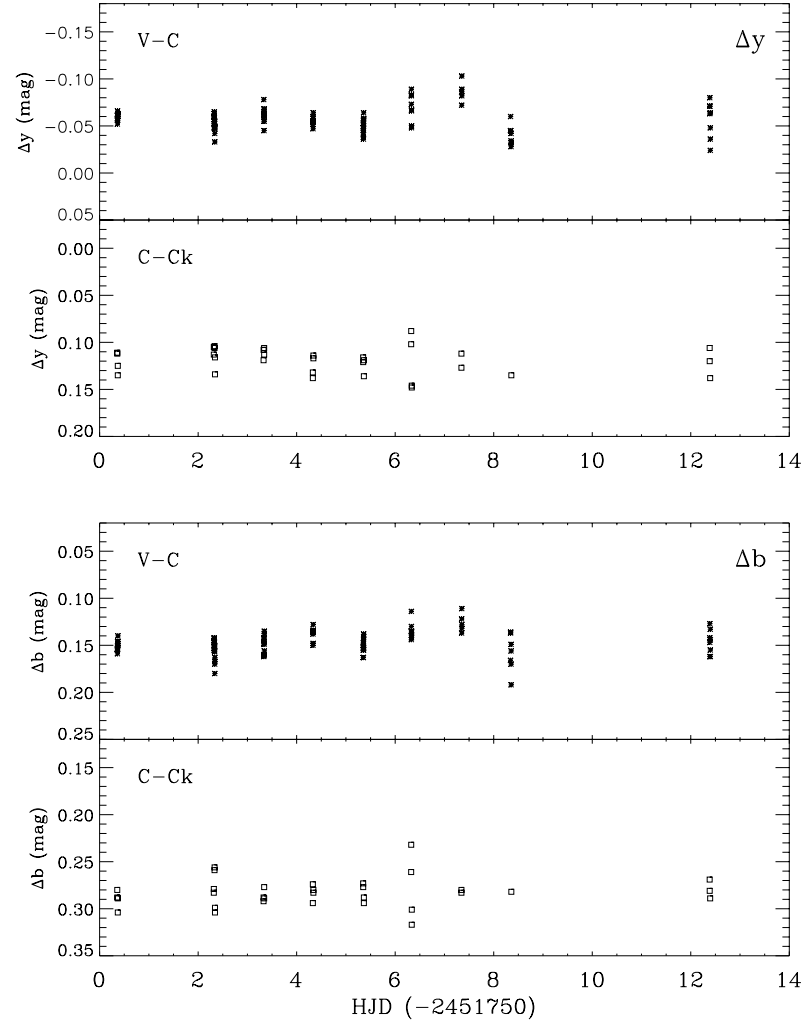

Fig. 2. $\Delta y$ magnitudes of HD 111456 and $\mathrm{C}-\mathrm{Ck}$ versus heliocentric Julian day (upper panels). $\Delta b$ magnitudes of HD 111456 and C-Ck (lower panels).

variations during the observing run. A variation of similar amplitude is also present in the Hipparcos magnitudes.

A search for periodicities in our data by applying the Discrete Fourier Transform (DFT) technique and the CLEAN algorithm (Roberts et al. 1986) did not give any evidence of a reliable period.

Our uvby photometry, averaging the 84 individual measurements, yields $V=5.826 \pm 0.016, b-y=0.320 \pm 0.012$, $m_{1}=0.155 \pm 0.019, c_{1}=0.346 \pm 0.026$. The values obtained by Crawford \& Barnes (1969) from an average of three measurements are $b-y=0.318, m_{1}=0.155, c_{1}=0.361$.

We used the $u v b y \beta$ procedure (Moon 1985) to estimate the effective temperature and the absolute magnitude and found $T_{\text {eff }}=6350 \pm 200 \mathrm{~K}$, and $M_{\mathrm{V}}=4 \cdot 2 \pm 0.2$. The absolute magnitude deduced by means of Hipparcos distance is instead $3.9 \pm 0.2$. This discrepancy could be due to the ultraviolet excess, affecting the Strömgren indices which leads to incorrect values of metallicity, $T_{\text {eff }}$ and $M_{\mathrm{V}}$. Such UV excess, with respect to older main-sequence stars, has been observed by Crawford \& Barnes (1969) and is confirmed by our analysis of the spectral distribution (see Sect. 4.2).

\subsection{Rotational velocity}

From the ELODIE spectra we made a new determination of $v \sin i$ by using the routine ROTFIT written by one of us (AF) in the IDL environment. This procedure derives the $v \sin i$ of an observed stellar spectrum by means of an iterative process 
on a spectral template, i.e. from the comparison of the target spectrum with that of a slow rotating star of the same spectral type, acquired with the same instrumental setup, progressively broadened by convolution with a rotational profile of increasing $v \sin i$.

The value of the rotational velocity for which the sum of residuals is minimum provides the $v \sin i$ of the target. From the ELODIE spectrum of HD 216835 used as a main template, we have obtained for HD $111456 v \sin i=41.5 \pm 1.1 \mathrm{~km} \mathrm{~s}^{-1}$, as an average value of many echelle orders to which ROTFIT was applied. We obtained similar results by using HD 181096 as template spectrum. By using Procyon (F5 IV, $T_{\text {eff }}=6650 \mathrm{~K}$ ) or $\mathrm{HD} 128429\left(\mathrm{~F} 5 \mathrm{~V}, v \sin i \simeq 14 \mathrm{~km} \mathrm{~s}^{-1}\right)$ we found $42.4 \pm$ $0.9 \mathrm{~km} \mathrm{~s}^{-1}$ and $40.3 \pm 2.0 \mathrm{~km} \mathrm{~s}^{-1}$, respectively. These values are fully consistent, within the errors, with the $v \sin i$ value found with the more appropriate template HD 216835, therefore we adopted $v \sin i=41.5 \pm 1.1 \mathrm{~km} \mathrm{~s}^{-1}$ as projected rotational velocity of HD 111456 .

The value we measured is indeed a little larger than that of $35 \mathrm{~km} \mathrm{~s}^{-1}$ reported by Soderblom \& Mayor (1993), but is in good agreement with the value of $39 \mathrm{~km} \mathrm{~s}^{-1}$ reported by Wolff \& Simon (1997).

In Fig. 3 we show the simulation of the spectrum of HD 111456 with that of HD 216385 broadened at $41.5 \mathrm{~km} \mathrm{~s}^{-1}$. Three spectral orders at blue, yellow and red wavelengths are displayed. The sum of residuals is also shown, as a function of $v \sin i$, in the insets of Fig. 3 .

\subsection{Radial velocity}

With the aim to check for the presence of a companion, we measured the radial velocity of HD 111456 on our OHP and OAC spectra.

The absolute radial velocity scale has been based on a primary RV standard star, namely Arcturus ( $v_{\text {helio }}=-5.2 \mathrm{~km} \mathrm{~s}^{-1}$, Duflot et al. 1995), which has been observed both at the OHP and OAC. Additional F-type stars, whose radial velocity has been checked against Arcturus, have been also observed and used as secondary standard RV stars.

In order to take advantage of all the available spectral region, we used the cross-correlation technique (e.g. Simkin 1974; Gunn et al. 1996). We have cross-correlated each spectral order of the ELODIE and OAC spectra of HD 111456 with the proper template, avoiding the orders acquired with a low $\mathrm{S} / \mathrm{N}$ ratio $(\lambda<4500 \AA$ for the ELODIE spectra) or heavily contaminated by telluric lines. Whatever RV template is used, we always find an asymmetric peak in the cross-correlation function (CCF) for all spectral orders. To verify if the asymmetry observed in HD 111456 could be attributed to an occasional instrumental error we applied the cross correlation procedure to HD 171834, a F3 V star observed with ELODIE in the same run, and we found a symmetric CCF.

Some evidence of asymmetry is also seen in the unblended lines of HD 111456 when observed at the resolution of ELODIE, and it is more evident at red and yellow wavelengths, as displayed in the two lowest panels of Fig. 3.
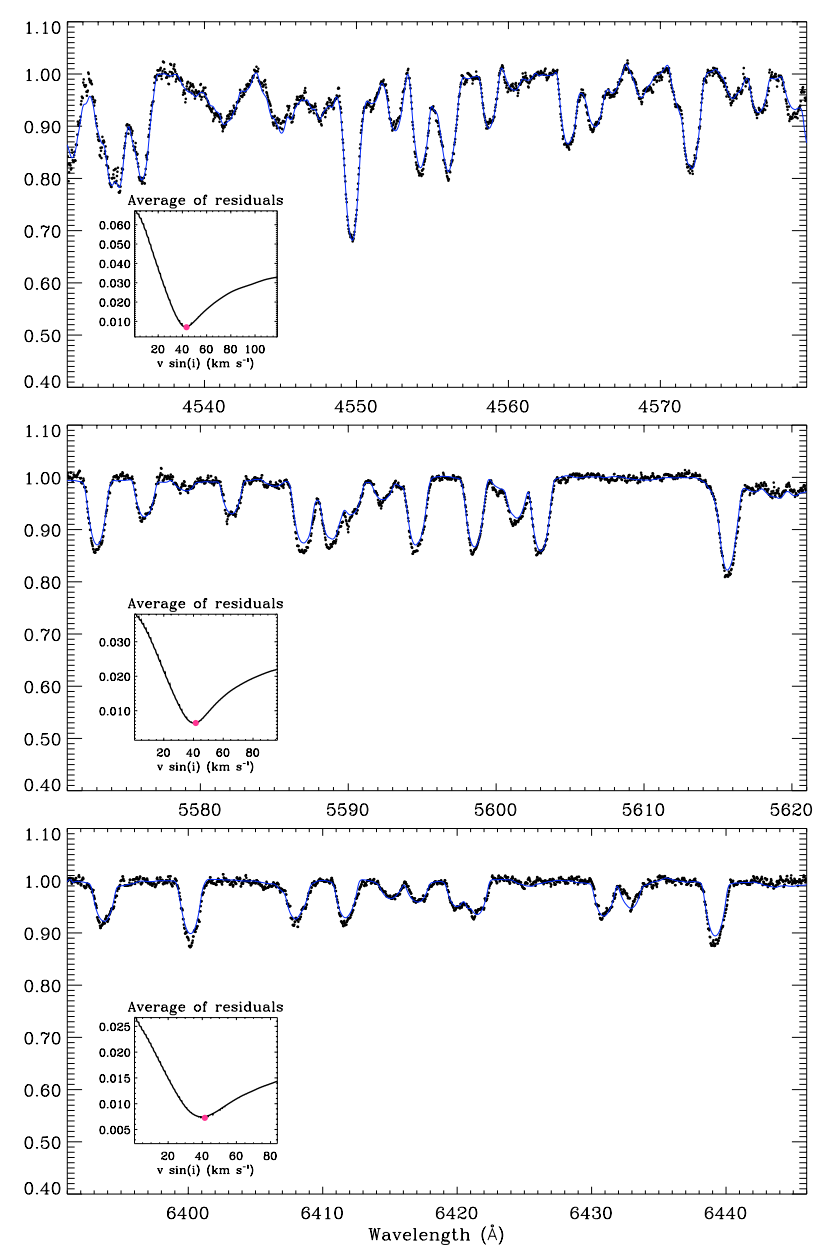

Fig. 3. Example of the simulation of HD 111456 spectrum (dots) with that of HD 216385 broadened at $41.5 \mathrm{~km} \mathrm{~s}^{-1}$ (thin lines) in three different spectral regions from blue to red wavelengths. The insets show the residuals as a function of template's $v \sin i$.

The CCF asymmetry appears constant over the time span of the OHP observations, but it shows a reverse behaviour two years later, in all the OAC data (Fig. 4). The CCF of the spectrum acquired in 2001 has a shape similar to those of 2000. A check for CCF shapes by using other stars observed at the OAC in the same run was made and no instrumental bias was found.

We have characterised the asymmetry of the CCF and spectral lines by means of its bisector (see e.g. Freire et al. 1977; Dravins 1987; Gray 1992; Dempsey et al. 1992). However, it is worth noticing that the bisector of HD 111456 in ELODIE spectra has a reversed C shape with respect to that of Procyon (Dravins 1987, 1990; Allende Prieto et al. 2002) and a much larger velocity span (5-8 $\mathrm{km} \mathrm{s}^{-1}$ for HD 111456 versus $0.5-1 \mathrm{~km} \mathrm{~s}^{-1}$ for Procyon). Then it seems unreasonable to invoke stellar granulation as the source of lines and CCF asymmetries in HD 111456, since it would require very large velocity fields in its atmosphere.

In the hypothesis that these $\mathrm{CCF}$ distortions are due to some physical effect intrinsically related to HD 111456 atmosphere, we have determined the centroid of the CCF by fitting a Gaussian to the overall peak. 


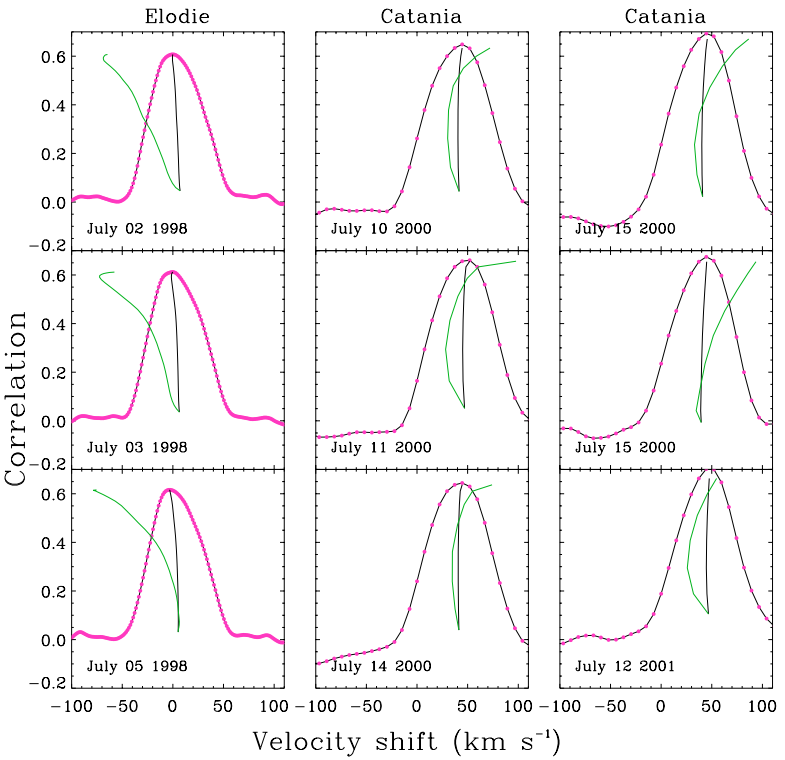

Fig. 4. Sample of CCFs. HD 216385 has been used as template for ELODIE spectra, while HD 181096 has been used for the OAC ones. The peak bisector is also shown in the plot scale (thick lines) and in a ten-times expansion (thin lines). Note the change in the asymmetry of CCF from 1998 to 2000.

In addition to this measurements we have tried to deconvolve, by a two-Gaussian fit, the asymmetric CCF peak as due to the overlap of two CCF peaks (two stars) very close in radial velocity.

We measured the RV by means of the CCFs of all useful orders and made the weighted averages for each spectrum.

Table 2 lists the average RV values of the centroid and of the two RV components deduced as described above, where 1 stays for the stronger peak component, together with the standard errors of the weighted means.

We have also evaluated the radial velocity of HD 111456 from two high-resolution IUE spectra retrieved from the IUE final archive (Nichols \& Linsky 1996). We used the IUE archive spectrum LWR09703 of Arcturus as template for the crosscorrelation that was performed as described in Frasca \& Lanza (2000). Table 2 contains also the RV values measured in the IUE spectra.

A RV variation, significant with respect to the standard errors, appears from Table 2. However, the full range of variation of $V_{\mathrm{c}}\left(\simeq 7 \mathrm{~km} \mathrm{~s}^{-1}\right)$ is comparable with the velocity span of the $\mathrm{CCF}$ bisectors. So, if the changing CCF asymmetry is not due to binarity, but to some other effect, this latter could be also responsible for the observed variation of $V_{c}$.

We have also found old RV measurements in the literature, though with errors larger than those of our data, and they span from -9.5 to $-18.4 \mathrm{~km} \mathrm{~s}^{-1}$ (Adams \& Joy 1923; Harper 1934; Roman 1949).

\subsection{Lithium abundance}

For HD 111456 we measured an equivalent width of the lithium $\lambda 6708$ line $W_{\mathrm{Li}}=0.023 \pm 0.005 \AA$, as an average value from the three ELODIE spectra, after subtracting a spectral template
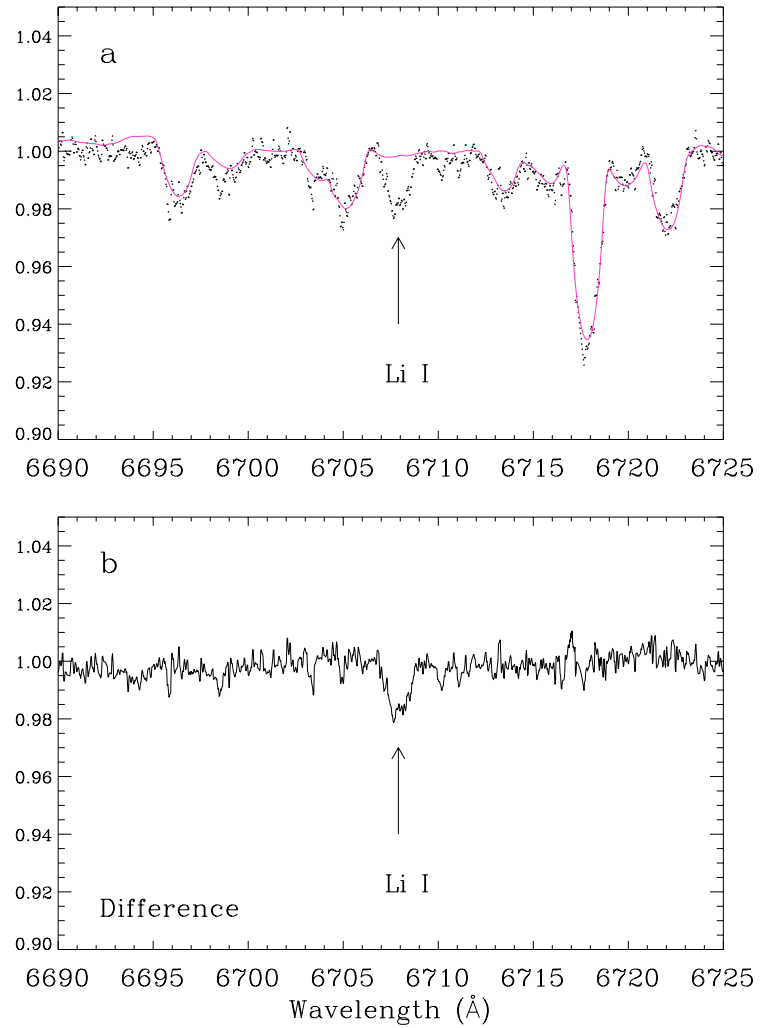

Fig. 5. a) The Li I $\lambda 6708$ region of HD 111456 as recorded with ELODIE (dots), with the superimposed spectral template (thin line). b) The residual spectrum, showing only the Li I absorption.

Table 2. Radial velocities of HD 111456.

\begin{tabular}{|c|c|c|c|c|}
\hline $\begin{array}{c}\text { HJD } \\
-2400000\end{array}$ & $\begin{array}{c}V_{\mathrm{c}} \\
\left(\mathrm{km} \mathrm{s}^{-1}\right)\end{array}$ & $\begin{array}{c}V_{1} \\
\left(\mathrm{~km} \mathrm{~s}^{-1}\right)\end{array}$ & $\begin{array}{c}V_{2} \\
\left(\mathrm{~km} \mathrm{~s}^{-1}\right)\end{array}$ & Instr. \\
\hline 44879.4508 & $-11.5_{ \pm 1.7}$ & - & - & IUE \\
\hline 45126.5800 & $-7.8_{ \pm 1.6}$ & - & - & “ \\
\hline 50983.3843 & $-5.8_{ \pm 2.1}$ & $-16.3_{ \pm 3.7}$ & $10.1_{ \pm 4.8}$ & AURELIE \\
\hline 50993.3856 & $-5.8_{ \pm 2.1}$ & $-18.5_{ \pm 2.9}$ & $9.0_{ \pm 2.8}$ & \\
\hline 50997.4428 & $-6.7_{ \pm 0.6}$ & $-19.8_{ \pm 0.7}$ & $7.7_{ \pm 0.8}$ & ELODIE \\
\hline 50998.3778 & $-6.7_{ \pm 0.7}$ & $-20.0_{ \pm 0.7}$ & $5.7_{ \pm 0.8}$ & “ “ \\
\hline 51000.3571 & $-6.5_{ \pm 0.7}$ & $-19.5_{ \pm 0.7}$ & $6.3_{ \pm 0.8}$ & “ $" ~ “$ \\
\hline 51736.4867 & $-13.3_{ \pm 0.7}$ & $-2.4_{ \pm 1.3}$ & $-33.4_{ \pm 1.5}$ & REOSC \\
\hline 51737.4418 & $-10.9_{ \pm 0.9}$ & $1.9_{ \pm 1.3}$ & $-31.1_{ \pm 2.1}$ & \\
\hline 51740.3843 & $-11.9_{ \pm 0.2}$ & $1.8_{ \pm 1.6}$ & $-30.6_{ \pm 0.8}$ & “ $،$ \\
\hline 51741.4058 & $-12.7_{ \pm 0.7}$ & $0.8_{ \pm 1.2}$ & $-32.0_{ \pm 2.8}$ & “ $،$ \\
\hline 51741.4521 & $-13.1_{ \pm 1.0}$ & $0.6_{ \pm 1.4}$ & $-29.1_{ \pm 1.3}$ & “ $،$ \\
\hline 52103.3996 & $-10.6_{ \pm 0.6}$ & - & - & “ \\
\hline
\end{tabular}

(Fig. 5b) like those obtained from the rotationally broadened ELODIE spectra of HD 181096 and HD 216385 in which the Li I line is not observed at all.

From the $W_{\mathrm{Li}}$ measured by us we have estimated the lithium abundance using both the Pavlenko \& Magazzù (1996) NLTE calculations and the LTE curves of growth reported by Soderblom et al. (1993b). A value of $N(\mathrm{Li}) \simeq 2.5$ is obtained 


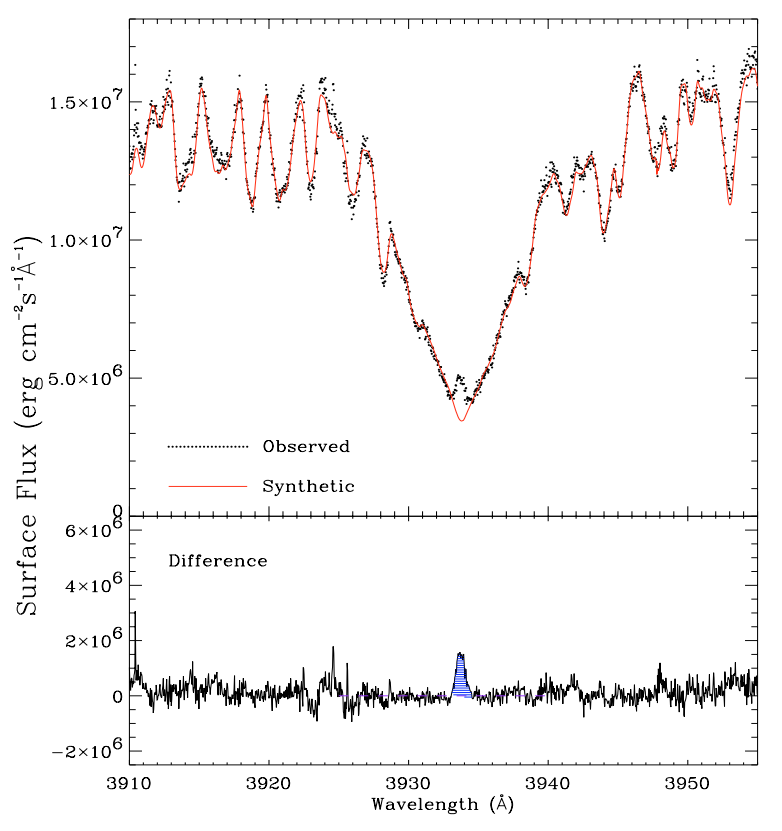

Fig. 6. Upper panel: The $\mathrm{K}$ line of an ELODIE spectrum of HD 111456 (dots). The photospheric template (HD 216385 broadened at $41.5 \mathrm{~km} \mathrm{~s}^{-1}$ ) is displayed superimposed by the thin line. Lower panel: The result of the subtraction emphasizing the residual core emission.

with both calibrations. This abundance is in excellent agreement with that derived by Soderblom et al. (1993a).

The $\mathrm{Li}$ abundance of HD 111456 is significantly smaller than that observed in Pleiades and $\alpha$ Per stars of similar spectral type (Soderblom et al. 1993a), and is consistent with the lithium dip observed for mid-F stars in intermediate-age clusters like Hyades and Coma, the so-called "lithium chasm" (Boesgaard \& Tripicco 1986). This feature is believed to be due to a lithium depletion mechanism operating during the first stages of the main-sequence lifetime. Several mechanisms, like mass-loss, diffusion and slow mixing have been proposed as the cause for the observed gap (e.g. Deliyannis et al. 1998). Anyway, such a value for $N(\mathrm{Li})$ indicates that the mechanism responsible for the Li depletion in mid-F stars has already been efficient enough to the age of 300-400 Myr of UMa group.

\subsection{Ca II and $\mathrm{Mg}$ II emission flux}

The observed Ca II H and $\mathrm{K}$ lines (Fig. 6, upper panel) show chromospheric asymmetric emission cores. To better evaluate the chromospheric emission flux in the cores of the Ca II lines we subtracted a photospheric template obtained from the spectrum of HD 216385, broadened to $v \sin i=41.5 \mathrm{~km} \mathrm{~s}^{-1}$. The very low activity level of HD 216385 is witnessed by the absence of emission in the cores of $\mathrm{Mg}$ II $\mathrm{h}$ and $\mathrm{k}$ lines (Fig. 7). This inactive template reproduces quite well both the photospheric spectrum and the Ca II line wings of HD 111456, suggesting a good spectral type match. A good agreement has also been obtained using HD 181096 as template.

By integrating the emission core residuals (Fig. 6, lower panel), we obtain an average value for the Ca II K chromospheric flux $F_{\mathrm{K}}=1.34 \times 10^{6} \pm 0.3 \times 10^{6} \mathrm{erg} \mathrm{cm}^{-2} \mathrm{~s}^{-1}$ and a

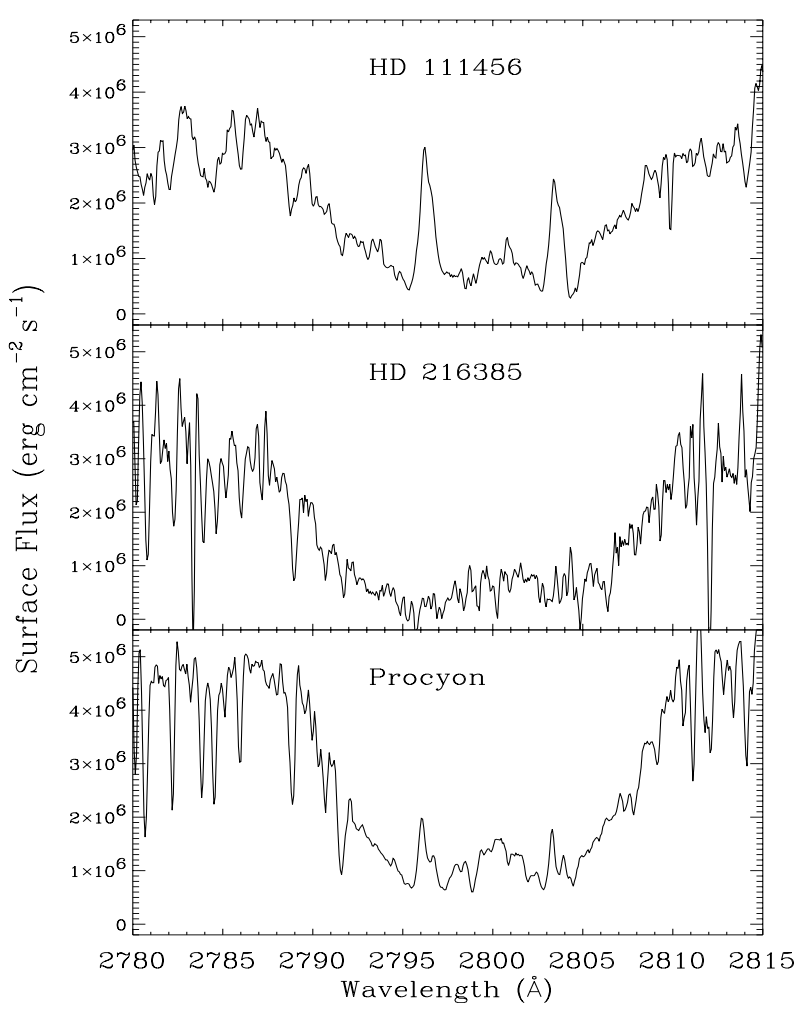

Fig. 7. The Mg II doublet as recorded by IUE at high resolution. The spectrum of HD 111456 (upper panel) shows a strong asymmetrical emission, whereas in HD 216385 (middle panel), if any, it is undetectable. The spectrum of Procyon (lower panel) displays instead a weak double-peaked emission.

ratio $F_{\mathrm{K}} / F_{\mathrm{H}} \simeq 1.3$. The Ca II $F_{\mathrm{K}}$ emission flux appears to be constant, within the errors, over the full observing season.

In addition, HD 111456 shows prominent chromospheric emission cores on $\mathrm{h}$ and $\mathrm{k} \mathrm{Mg}$ II lines ( $\lambda 2795$ and $\lambda 2802 \AA)$ as seen in two high-resolution IUE spectra taken in October 1981 and June 1982 and retrieved from the IUE Final Archive (Nichols \& Linsky 1996) and the INES database (2000).

In Fig. 7 we compare the UV flux at the stellar surface in the Mg II region of the inactive star HD 216385 with that of HD 111456 which displays strong asymmetric emission cores and with that of Procyon showing an intermediate case between the two other stars.

We can estimate the chromospheric flux in the Mg II lines by integrating the $\mathrm{h}$ and $\mathrm{k}$ emission cores between the $\mathrm{k}_{1}$ and $\mathrm{h}_{1}$ features. This yields $F_{\mathrm{k}}=2.8 \times 10^{6} \mathrm{erg} \mathrm{cm}^{-2} \mathrm{~s}^{-1}$ in both spectra, higher than the upper limit of the flux displayed by the late F-type stars (Blanco et al. 1982). We also measure a flux ratio $F_{\mathrm{k}} / F_{\mathrm{h}}=1.2$.

For mid-F Hyades stars, recent works based on IUE and HST/STIS observations, (Bohm-Vitense et al. 2001, 2002) show that, for stars with $v \sin i$ from 20 to $40 \mathrm{~km} \mathrm{~s}^{-1}$, the $\mathrm{Mg}$ II $F_{\mathrm{k}} \leq 1.6 \times 10^{6}$, i.e. almost one half the value we found for HD 111456. These works also give an average value of $F_{\mathrm{k}} / F_{\mathrm{h}}$ of about 1.5 which points toward a lower optical depth at the line center for Hyades stars with respect to HD 111456. 


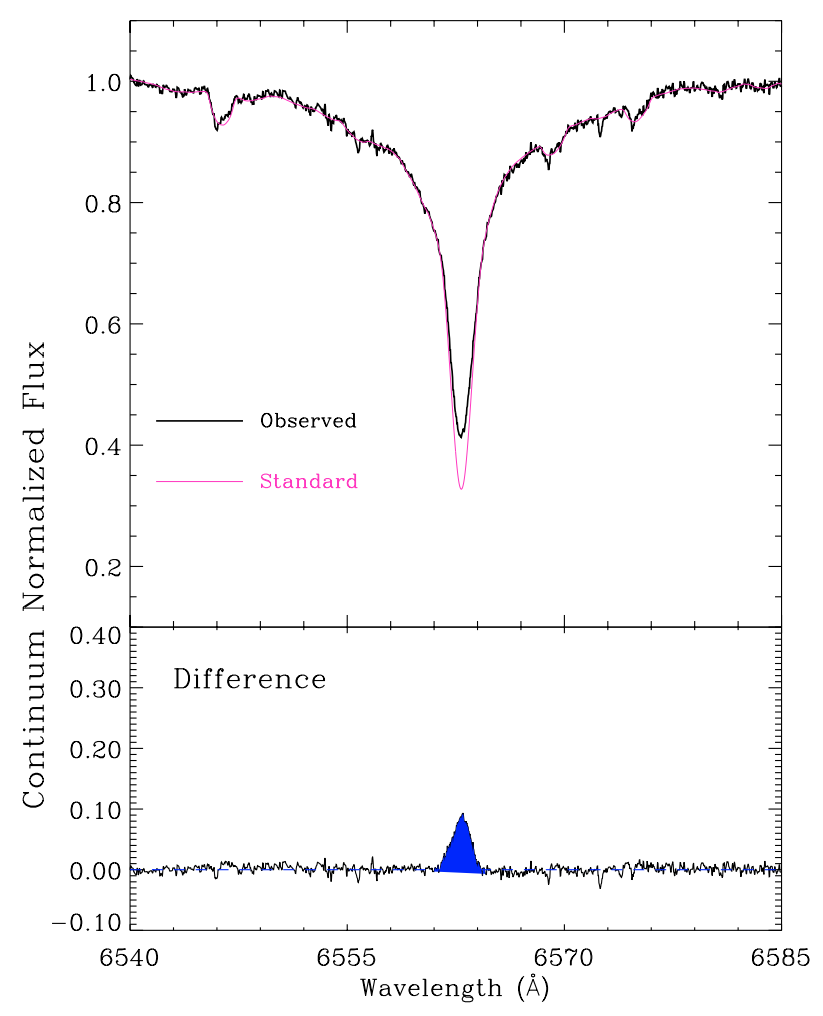

Fig. 8. An $\mathrm{H} \alpha$ profile of HD 111456 (thick line) with the superimposed rotationally broadened spectrum of the standard star HD 216385 (thin line). Low drawing shows the difference of both spectra and the $\mathrm{H} \alpha$ core filled in by chromospheric emission.

\subsection{The $H \alpha$ and $H \beta$ lines}

We evaluated the $\mathrm{H} \alpha$ chromospheric emission by means of the "spectral synthesis" technique (Paper I) using the spectrum of HD 216385, convolved with a rotation profile of $v \sin i=41.5 \mathrm{~km} \mathrm{~s}^{-1}$, as inactive template. As in the case of the $\mathrm{Ca}$ II lines, this template matches much better the $\mathrm{H} \alpha$ line wings than the spectrum of Procyon, which has a slightly larger $T_{\text {eff }}$.

In Fig. 8 an ELODIE spectrum of HD 111456 in the $\mathrm{H} \alpha$ region is shown (thick line) together with the rotationally broadened template (thin line). A significant positive peak above the noise is clearly seen in the difference spectrum.

The same procedure was used for the analysis of the spectra acquired at the OAC.

The net $\mathrm{H} \alpha$ equivalent width, $W_{\mathrm{H} \alpha}$, has been measured (Table 3 ) in such residual spectra by integrating the net emission profile (hatched area in Fig. 8).

We note that the $W_{\mathrm{H} \alpha}$ and $\mathrm{H} \alpha$ flux values are not very different over each season, but all the OAC data acquired in 2000, though within the errors, are systematically higher (about 20\%) than the OHP ones. These small differences could be due to the different resolution and instrumental setup. However, from the spectrum obtained at the OAC in 2001 we obtain a value equal to that measured in 1998 on the ELODIE spectra, and this could be an argument in favour of little changes of the chromospheric activity level on long time scales.

$W_{\mathrm{H} \alpha}$ has been converted into $\mathrm{H} \alpha$ surface flux (Table 3) multiplying it by the flux at $\mathrm{H} \alpha$ continuum, $F_{\mathrm{c}}(\mathrm{H} \alpha)=1.1 \times$ $10^{7} \mathrm{erg} \mathrm{cm}^{-2} \mathrm{~s}^{-1} \AA^{-1}$ (see Sect. 4.1).

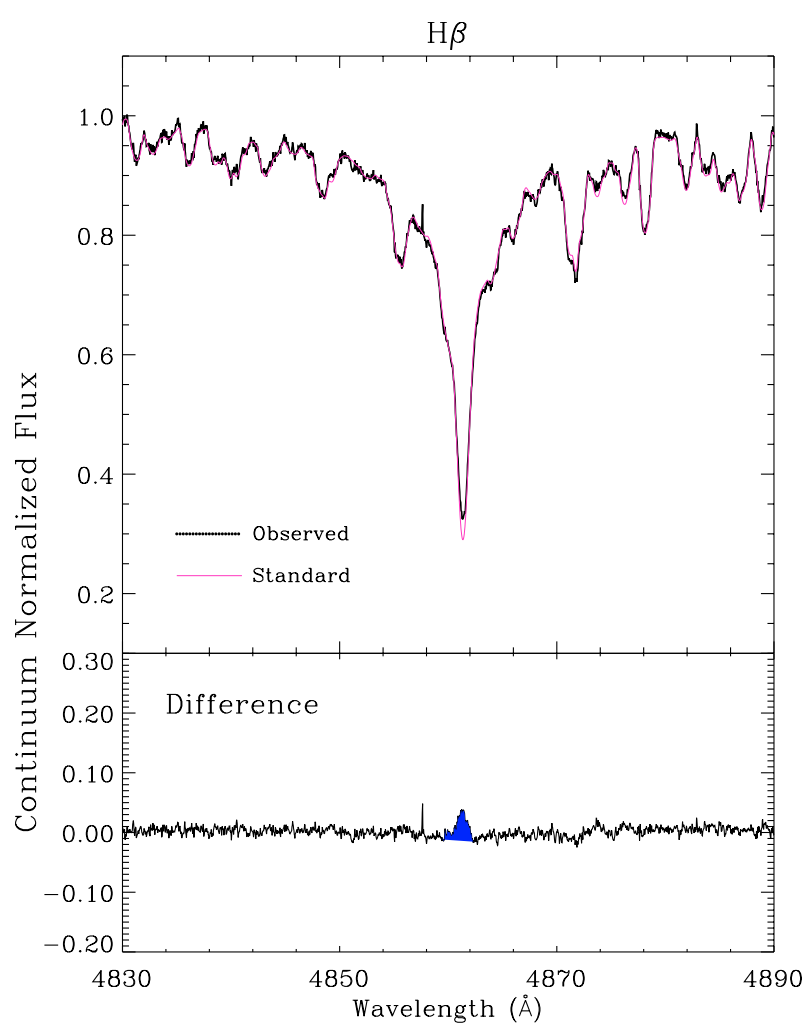

Fig. 9. The $\mathrm{H} \beta$ line of HD 111456 as recorded with ELODIE (thick line). The inactive template (HD 216835 broadened at $41.5 \mathrm{~km} \mathrm{~s}^{-1}$ ) is shown with a thin line for comparison. The residual flux, showing a small emission peak in the line core, is displayed in the lower panel.

Table 3. $\mathrm{H} \alpha$ equivalent widths.

\begin{tabular}{ccccl}
\hline \hline $\begin{array}{c}\text { Hel. Jul. Day } \\
-2400000\end{array}$ & $\begin{array}{c}W_{\mathrm{H} \alpha} \\
(\AA)\end{array}$ & $\begin{array}{c}\text { error } \\
(\AA)\end{array}$ & $\begin{array}{c}F_{\mathrm{H} \alpha} \\
\left(10^{6} \mathrm{erg} \mathrm{cm}^{-2} \mathrm{~s}^{-1}\right)\end{array}$ & Instr. \\
\hline 50997.4428 & 0.130 & 0.021 & 1.43 & ELODIE \\
50998.3778 & 0.130 & 0.021 & 1.43 & ““ ““ \\
51000.3571 & 0.140 & 0.020 & 1.54 & “ \\
51736.4867 & 0.161 & 0.041 & 1.77 & REOSC \\
51737.4418 & 0.153 & 0.052 & 1.68 & ““ ““ \\
51740.3843 & 0.156 & 0.044 & 1.72 & “ “ “ \\
51741.4058 & 0.177 & 0.044 & 1.95 & “ “ \\
51741.4521 & 0.167 & 0.046 & 1.84 & “ “ \\
52103.3996 & 0.127 & 0.038 & 1.40 & \\
\hline
\end{tabular}

We also observed a slightly filled-in $\mathrm{H} \beta$ line in the ELODIE spectra. The rotationally-broadened template (HD 216385) gives a perfect reproduction of all the spectral region around $\mathrm{H} \beta$ (Fig. 9). The resulting filling of the line core has a net equivalent width of $W_{\mathrm{H} \beta}=0.024 \pm 0.016 \AA$.

The $\mathrm{H} \beta$ line flux, $F_{\mathrm{H} \beta}=3.9 \times 10^{5} \mathrm{erg} \mathrm{cm}^{-2} \mathrm{~s}^{-1}$, has been derived from $W_{\mathrm{H} \beta}$ as for the $\mathrm{H} \alpha$ line, using the corresponding continuum flux $F_{\mathrm{c}}(\mathrm{H} \beta)=1.6 \times 10^{7} \mathrm{erg} \mathrm{cm}^{-2} \mathrm{~s}^{-1} \AA^{-1}$. On the basis of the $\mathrm{H} \alpha$ and $\mathrm{H} \beta$ flux we evaluated a Balmer decrement $F_{\mathrm{H} \alpha} / F_{\mathrm{H} \beta} \simeq 4 \pm 2$. As suggested by Hall \& Ramsey (1992), high values of the Balmer decrement are typical of solar prominences seen against the disk, whereas solar plages or flares have 

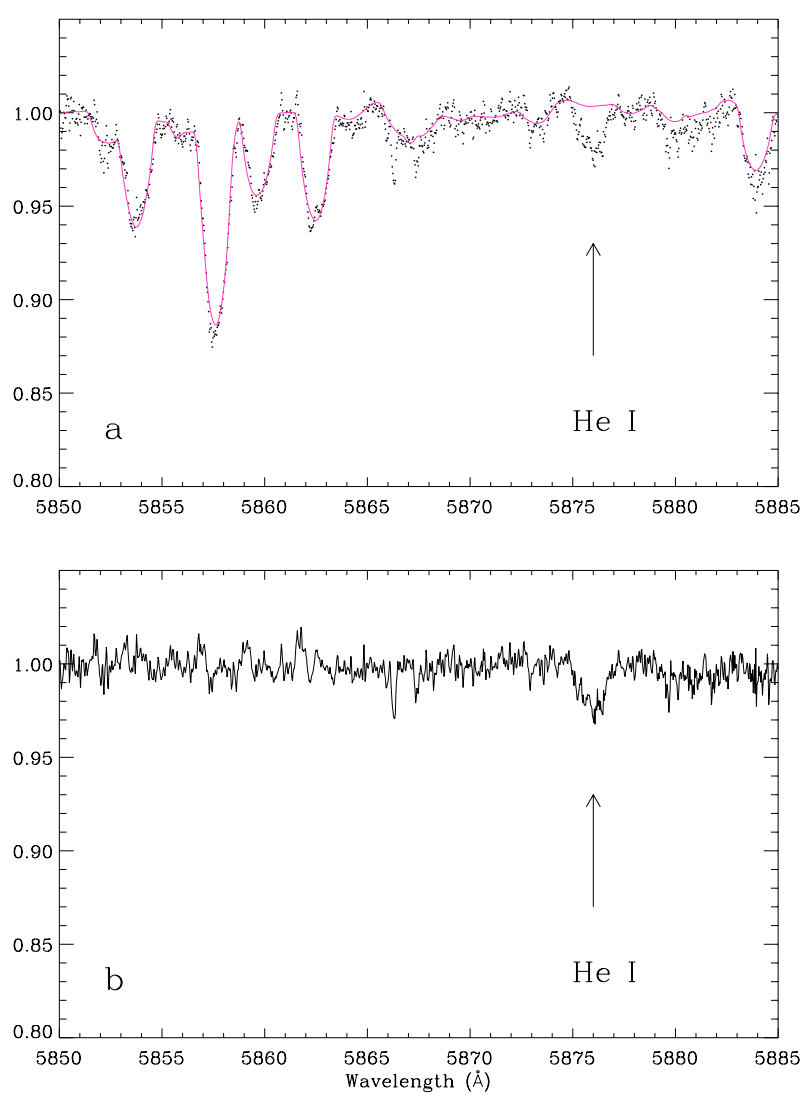

Fig. 10. a) The He I $\lambda 5876$ region of HD 111456 as recorded with ELODIE (dotted line). The inactive template is shown with a thin continuous line for comparison. b) The residual spectrum, which emphasizes the $\mathrm{D}_{3}$ absorption.

always values of about 1-2. The prominence models developed by Heasley \& Mihalas (1976) lead to high Balmer decrement values, ranging from 5 to 12 . A theoretical NLTE model by Buzasi (1989) confirms the same picture.

The Balmer decrement $F_{\mathrm{H} \alpha} / F_{\mathrm{H} \beta} \simeq 4$ would suggest that the activity structures in HD 111456 are more similar to solar prominences or, if related to the chromosphere, they should have physical conditions quite different with respect to solar plages.

\subsection{He I $\lambda 5876$ line}

The He I $\mathrm{D}_{3}$ line at $5876 \AA$ is an absorption feature and represents a good chromospheric indicator for F spectral-type stars (Garcia Lopez et al., 1993). This indicator sets the limit for the onset of magnetic activity at $B-V=0.42$. The inactive stars we used as references did not show any evidence of He I absorption.

We used the average of the three ELODIE spectra for a better definition of the $\mathrm{He} \mathrm{I}_{3}$ line (Fig. 10) and we obtain an equivalent width $W_{\mathrm{HeI}}=37 \pm 5 \mathrm{~m} \AA$, which is in very good agreement with the value of $36 \pm 5 \mathrm{~m} \AA$ given by Garcia Lopez et al. (1993).

\section{Discussion}

\subsection{The spectral energy distribution}

We evaluated the flux emitted in the optical and IR regions by HD 111456 from the $U B V R I$ (Johnson et al. 1966),

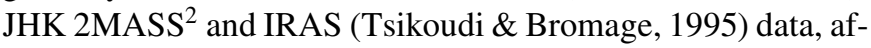
ter a standard dereddening (Cox 2000). However, the reddening of HD 111456 is practically negligible in near all photometrical bands.

These observed fluxes were compared with those obtained by integrating, into the proper pass-bands, the synthetic spectra generated with the NextGen (Hauschildt et al. 1999a,b) photospheric models.

The model with $T_{\text {eff }}=6400 \mathrm{~K}, \log g=4.5$ and a stellar radius $R=1.22 R_{\odot}$ gives the best agreement with the observed spectral energy distribution (SED). Taking into account the distance of $24 \mathrm{pc}$ (Hipparcos), these values are fully compatible with a F5-6 main sequence star.

Furthermore, the stellar radius we deduce is in good agreement with the value of $1.28 R_{\odot}$ derived by means of the BarnesEvans relation (Barnes \& Evans 1976) which gives the angular diameters for late-type stars.

In Fig. 11 we show the comparison of the observed flux at Earth (asterisks), the flux computed from the model (triangles) and the synthetic spectrum (full line). Linear and logarithmic scales have been used in the two plots to give a better view in different spectral ranges.

The colour excess at $U$ wavelength is consistent with the ultraviolet excess found by Crawford \& Barnes (1969) in late F stars of the Ursa Major group and ascribed to facular activity. Measured IR fluxes at 60 and $100 \mu \mathrm{m}$ are significantly above the flux predicted by the model. This disagreement may be due to interstellar or circumstellar matter, or could be due to a lower accuracy of the model at these wavelengths or to some problem of IRAS calibration. Similar discrepancies in far-IR fluxes are also seen in other late F-dwarf stars with low activity.

\subsection{Is the HD 111456 activity due to binarity?}

The RV variations we observed suggest the possibility that HD 111456 could be binary. We made some tests of this hypothesis by simulating the influence of an eventual companion on the observed spectra at different wavelengths.

\subsubsection{Synthesis of the observed SED with a composite spectrum}

Firstly we produced a combined synthetic spectrum of the presumed binary by co-adding two NextGen spectra, one of a F-type star and one of a cool K-M dwarf. The best solution we found is represented by a model of a hot star with $T_{\text {eff }}=6600 \mathrm{~K}$ and radius $r_{\mathrm{hot}}=1.13 R_{\odot}$, corresponding to a F3-4V spectral

\footnotetext{
2 2MASS (Two Micron All Sky Survey) is a joint project of the University of Massachusetts and the Infrared Processing and Analysis Center/California Institute of Technology, funded by the National Aeronautics and Space Administration and the National Science Foundation.
} 

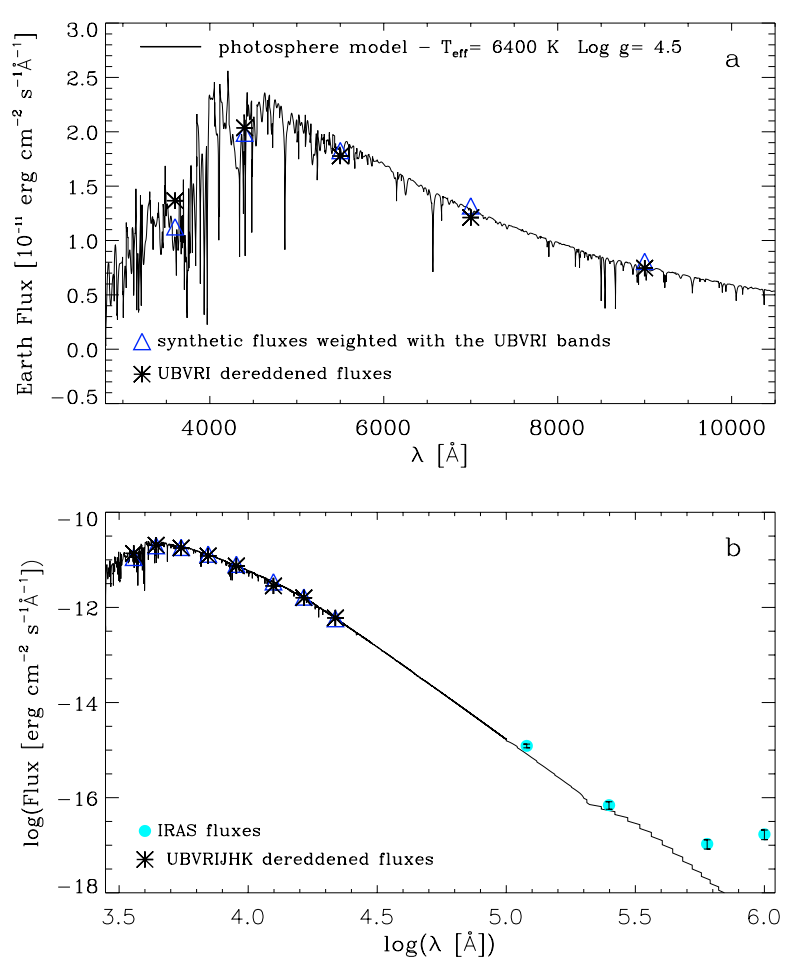

Fig. 11. a) The spectral distribution of HD 111456 as deduced from UBVRIJHK observed magnitudes (asterisks) compared with the synthetic fluxes (triangles) obtained by integrating in the proper passbands a synthetic NextGen model (full line) for $T_{\text {eff }}=6400 \mathrm{~K}$. b) The same figure is shown in a log-log scale, where the $J H K$ and IRAS 12, 25, 60 and $100 \mu \mathrm{m}$ fluxes are also displayed.

type, and a cool-star spectrum with $T_{\text {eff }}=4400 \mathrm{~K}(\mathrm{~K} 5 \mathrm{~V})$ and a radius $r_{\text {cool }}=0.65 R_{\odot}$. As shown in Fig. 12 , no evident improvement in the fit of the observed SED with respect to the single-star case (Fig. 11) is obtained. Furthermore, in this case the hot stellar radius is not consistent with its spectral type, since a F3-4V star should have a radius of about $1.4 R_{\odot}$.

\subsubsection{Synthesis of Call lines with a composite spectrum}

We have tried to reproduce the observed Ca II lines with a composite spectrum built up with the sum of the rotationally broadened spectrum of HD 216385 (F-star) and a spectrum of an active $\mathrm{K} 4 \mathrm{~V}$ star belonging to the same moving group, i.e. HD 131156B, retrieved from the library of UES spectra of latetype stars (Montes \& Martin 1998). The observed Ca II spectrum of HD 111456 is fairly well reproduced, for a relative weight of the hot to cool component $F_{\text {hot }} / F_{\text {cool }}=25$, but this would require a cool component with a radius of about $1.5 R_{\odot}$. However, this flux ratio is not consistent with the value $F_{\text {hot }} / F_{\text {cool }} \simeq 128$ derived from the synthesis of the observed SED at these wavelengths.

In addition, we have explored the extreme case of a very active cool component, like a BY Dra or a Pleiades star. Two very active single-lined RS CVn binaries with an effective temperature comparable to that of HD 131156B are HU Vir (K0 III-IV) and BM CVn (K1 III), whose spectra in the Ca II region are
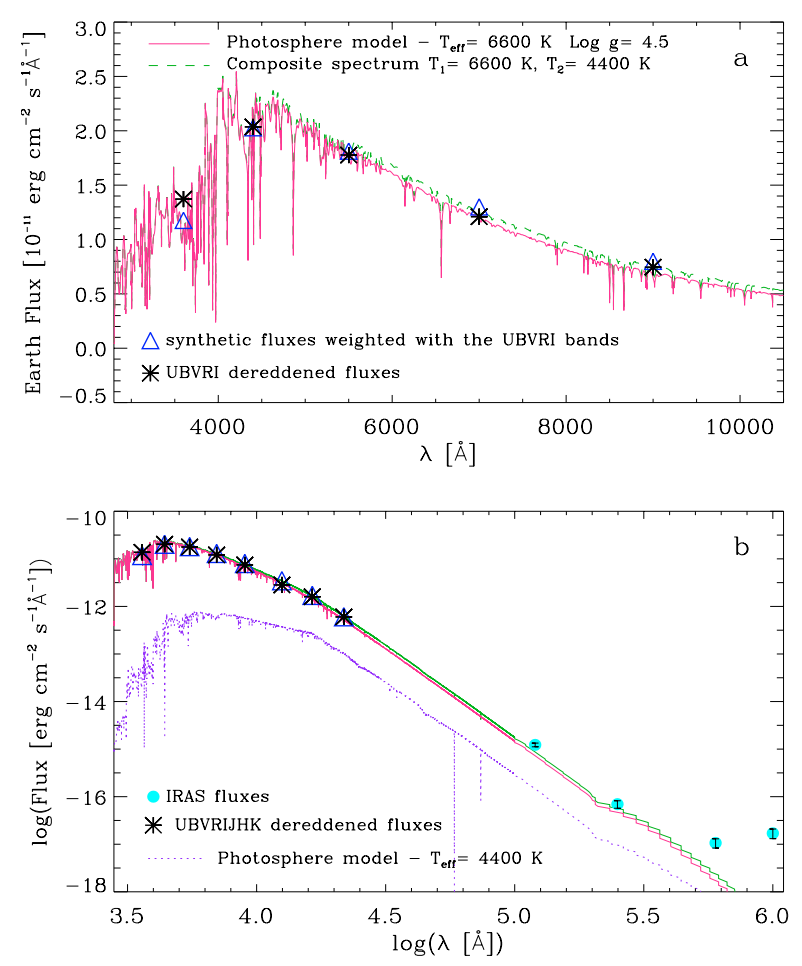

Fig. 12. Spectral distribution of HD 111456 as deduced from UBVRI magnitudes (asterisks) compared with a combination of two synthetic NextGeneration models for $T_{\mathrm{eff}}=6600 \mathrm{~K}$ and $4400 \mathrm{~K}$.

displayed by Montes et al. (2000). The peak intensity of the $K$ emission reaches 2.5 and 3.5 times the continuum level for $\mathrm{BM} \mathrm{CVn}$ and HU Vir, respectively, i.e. less than twice that of HD 131156B. Even in this case, we could fit the emission core observed in the Ca II lines of HD 111456 with a flux ratio $F_{\text {hot }} / F_{\text {cool }} \leq 40$, which is again not consistent with the value of 128 coming from the synthetic SED.

The same occurs when we try to reproduce other chromospheric lines, like $\mathrm{Mg}$ II, $\mathrm{H} \alpha, \mathrm{H} \beta$ and $\mathrm{He} \mathrm{I}_{3}$. We argue then that the observed activity signs are truly due to the $\mathrm{F}$ star.

On the other hand, the clear evidence of the $\mathrm{He} \mathrm{I}_{3}$ absorption line (Fig. 10) is strongly indicative of activity in a F-type star (Garcia Lopez 1993).

\subsection{Activity in F-type stars and HD 111456}

The strong activity of HD 111456 at chromospheric, TR and coronal levels, is consistent with its high rotation rate. On the one hand, another mid-F star belonging to UMa group, namely HD 125451 (F5 IV), has a rotational velocity ( $v \sin i=$ $46.0 \mathrm{~km} \mathrm{~s}^{-1}$, De Medeiros \& Mayor 1999) very close to that of HD 111456 and exhibits a comparable coronal activity level $\left(\log L_{X}=29.42\right)$. On the other hand, Schmitt et al. (1990) found a F5 V star in UMa group with a much lower X-ray luminosity (HD 38393, $\log L_{\mathrm{X}}=27.56$ ) as well as a lower rotational velocity ( $v \sin i=7.7 \mathrm{~km} \mathrm{~s}^{-1}$ Reimers et al. 2003).

Micela et al. (1996) found a similar behaviour in Pleaides late-F type stars, for which $\log L_{X}$ ranges from 29.1 to 29.9 and $v \sin i$, from 20 to $80 \mathrm{~km} \mathrm{~s}^{-1}$, with a clear increasing trend of X-ray activity with the rotational speed. For F stars 


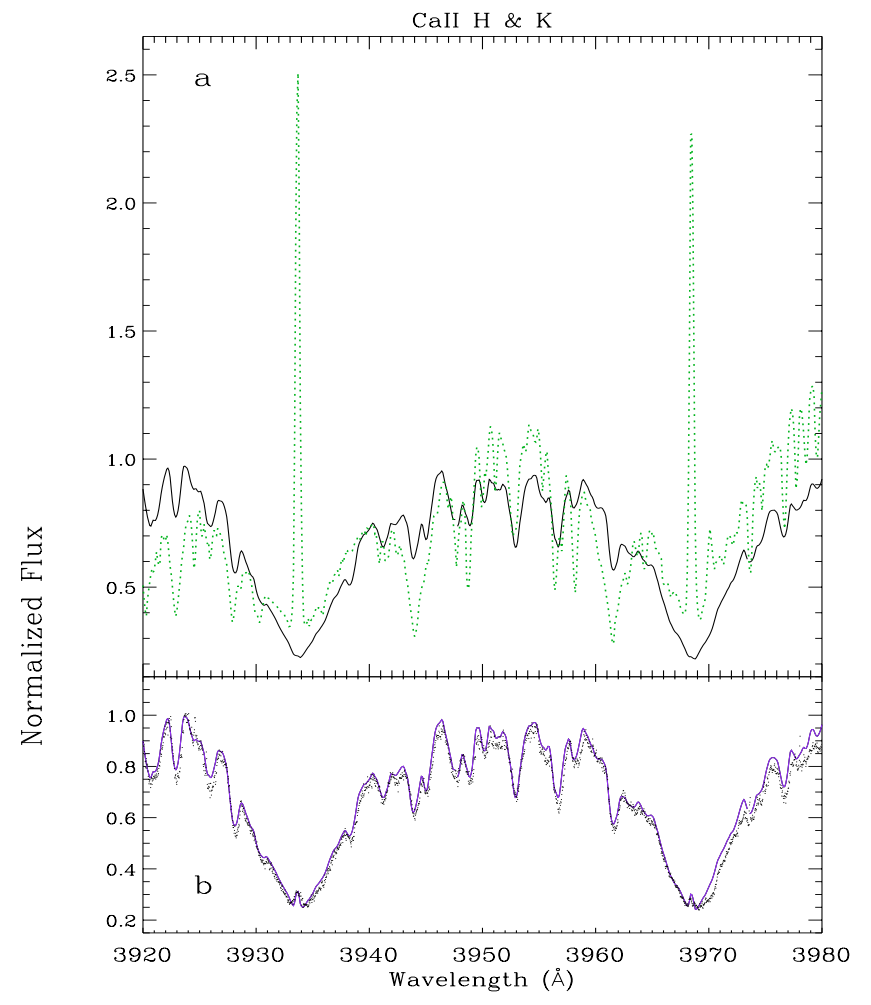

Fig. 13. a) The Ca II spectrum of HD $131156 \mathrm{~B}$ (dotted line) and the composite spectrum (solid line) obtained with the flux ratio $F_{\text {hot }} / F_{\text {cool }}=128$. No emission appears in the line cores. b) Simulation of the CaII spectral region (continuous line) with a flux ratio $F_{\text {hot }} / F_{\text {cool }}=25$, to which the real spectrum of HD 111456 (dots) has been superimposed.

belonging to $\alpha$ Per cluster, Prosser et al. (1996) found an average $\log L_{\mathrm{X}}$ about twice as high as that of Pleiades. Furthermore, all $\alpha$ Per members with $v \sin i>50 \mathrm{~km} \mathrm{~s}^{-1}$ show saturated levels of coronal activity, while stars with $v \sin i$ lower than the upper limit of $15 \mathrm{~km} \mathrm{~s}^{-1}$, show a wide range of coronal activity. For Hyades cluster, late-F type stars also exhibit X-ray and TR emission increasing with $v \sin i$ (Bohm-Vitense et al. 2002) in the range from 15 to $50 \mathrm{~km} \mathrm{~s}^{-1}$, while the early F-type stars in their sample seem not to follow this trend.

In this context we must recognize that magnetohydrodynamic mechanisms are the most efficient ones in heating the outer atmospheres of young late-F stars similar to HD 111456. While the hydrogen and helium convection zones are separated for early-F stars, they merge for slightly cooler F stars, where diffusion and evolution time scales are comparable and where an increase of the Rosseland opacity leads to an increase in the depth of the convection zone by factors of 3-5 (Turcotte et al. 1998). This can explain a delayed onset of an efficient dynamo able to strongly enhance the magnetic activity in young $F$ stars. At this point, the magnetic braking becomes effective and starts to carry away a good amount of angular momentum.

Anyway, the contemporaneous presence of fast and slow rotators in coeval late-F stars could be explained by the different initial angular momentum of each star on which the braking mechanism begins to work when the convection zone is sufficiently developed by the increasing opacity. So HD 111456 is representative of an initial fast-rotating stage where the dynamo works from a time not yet sufficient to produce a relevant magnetic braking.

\section{Conclusions}

We have made a spectroscopic and photometric study of HD 111456, one of the most active main-sequence mid-F type stars. In spite of its high activity degree, the photometry has not shown any evidence of rotational modulation, although some scattered variation at a very low amplitude level $\left(\leq 0^{\mathrm{m}} .03\right)$ could have been observed. The Strömgren photometry has given some evidence of an UV excess that can be related to facular activity.

By means of the spectral synthesis we have confirmed a spectral type of F5-6 and we have found a $v \sin i=$ $41.5 \mathrm{~km} \mathrm{~s}^{-1}$, in agreement with previous determinations. We have detected the Li I $\lambda 6708$ line confirming that HD 111456 is a young star and the lithium abundance is consistent with the Li gap observed at mid-F stars in intermediate-age clusters. This suggests that the strong lithium depletion in mid-F stars is already active at an age of 300-400 Myrs.

In addition, we have also detected emission cores in the $\mathrm{Ca}$ II $\mathrm{H}$ and $\mathrm{K}$ lines and a filling of both $\mathrm{H} \alpha$ and $\mathrm{H} \beta$ lines. We could also estimate the Balmer decrement $F_{\mathrm{H} \alpha} / F_{\mathrm{H} \beta} \simeq 4$ which could be indicative of extended matter of the kind of solar prominences.

The $\mathrm{He} \mathrm{I}_{3}$ absorption line confirms the strong activity of HD 111456. A very high chromospheric emission level is also indicated by the $h_{2}$ and $k_{2}$ emission features of $\mathrm{Mg}$ II in high resolution IUE spectra, whose net flux overwhelms the upper limits displayed by late F-type stars.

No rotational modulation of the $\mathrm{H} \alpha$ and $\mathrm{Ca}$ II flux has been observed, but some indication of long-term variations is suggested by our data. The non-detection of rotational modulation neither at chromospheric nor at photospheric level could be explained by a homogeneous distribution of active regions or by a very low inclination of the rotation axis with respect to the line of sight.

The anomalously high level of activity for a mid-F type star displayed by various chromospheric diagnostics leads to suspect that HD 111456 could in reality be a binary for which most of the observed activity arises from a cool active companion. Although the analysis of the radial velocities obtained with the cross-correlation technique applied to the whole sample of our spectra, has given some evidence of RV variations and has shown a changing asymmetric CCF peak, ascribable to a close binary system, the possibility of a composite spectrum is not supported by the spectral energy distribution (SED) analysis and by the synthesis of the observed Ca II profiles with composite spectra neither in the case of a young active cool star nor in the case of an evolved (giant or sub-giant) cool component like those of the very active RS CVn-type binaries.

On the other hand, the observed spectral energy distribution, from UV to IR wavelengths, is very well fitted by a synthetic spectrum of a single star with $T_{\text {eff }}=6400 \mathrm{~K}, \log g=4.5$ (spectral type F5-6 V). 
So, the strong activity in HD 111456 should be typical of a young mid-F star where high angular momentum has not yet been dissipated by magnetic braking.

An ultimate explanation of the behaviour and nature of HD 111456 will be possible only with further observations, both photometric and spectroscopic at medium and high resolution, spanning on a large temporal scale.

Acknowledgements. This work has been supported by the Agenzia Spaziale Italiana (contract no. I/R/111/01) and by the Regione Sicilia which are gratefully acknowledged. We would like to acknowledge the valuable support of the technical staff of Catania (OAC) and Haute Provence (OHP) observatories. During most of the time of this work AF and EM were visiting scientists at the Strasbourg Observatory whose support is gratefully acknowledged. We would like to thank the anonymous referee for his/her helpful comments and suggestions. This research has made use of the SIMBAD database, operated at CDS, Strasbourg, France, and of the General Catalogue of Photometric Data (GCPD) operated at the University of Lausanne, Switzerland.

\section{References}

Adams, W. S., \& Joy, A. H. 1923, ApJ, 57, 149

Allende Prieto, C. A., Asplund, M., García López, R. J., \& Lambert, D. L. 2002, ApJ, 567, 544

Baliunas, S. L., Horne, J. H., Porter, A., et al. 1985, ApJ, 294, 310

Baliunas, S. L., Donahue, R. A., Soon, W., et al. 1995, ApJ, 438, 269

Baranne, A., Queloz, D., Mayor, M., et al. 1996, A\&AS, 119, 373

Barnes, T. G., \& Evans, D. S. 1976, MNRAS, 174, 489

Blanco, C., Bruca, L., Catalano, S., \& Marilli, E. 1982, A\&A, 115, 280

Bohm-Vitense, E., Mena-Werth, J., Carpenter, K. J., \& Robinson, R. D. 2001, ApJ, 550, 457

Bohm-Vitense, E., Robinson, R. D., Carpenter, K. J., \& Mena-Werth, J. 2002, ApJ, 569, 941

Boesgaard, A. M., \& Tripicco M. J. 1986, ApJ, 302, L49

Buzasi, D. L. 1989, Ph.D. Thesis, Pennsylvania State Univ.

Cayrel, R., Cayrel de Strobel, G., Campbell, B., et al. 1983, A\&A, 123,89

Chupina, N. V., Reva, V. G., \& Vereshchagin, S. V. 2001, A\&A, 371, 115

Crawford, D. C., \& Barnes, J. V. 1969, AJ, 74, 407

Cox, A. N. 2000, Allen's Astrophysical Quantities (Springer-Verlag)

Deliyannis, C. P., Boesgaard, A. M., Stephens, A., et al. 1998, ApJ, 498, L147

De Medeiros, J. R., \& Mayor, M. 1999, A\&AS, 139, 433

Dempsey, R. C., Bopp, B. W., \& Strassmeier, K. G., et al. 1992, ApJ, 392,187

Donahue, R. A., Saar, S. H., \& Baliunas, S. L. 1996, ApJ, 466, 384

Dravins, D. 1987, A\&A, 172, 211

Dravins, D. 1990, A\&A, 228, 218

Duflot, M., Figon, P., \& Meyssonnier, N. 1995, A\&AS, 114, 269

Eggen, O. J. 1992, AJ, 103, 1302

Frasca, A., \& Lanza, A. F. 2000, A\&A, 356, 267

Frasca, A., Freire Ferrero, R., Marilli, E., \& Catalano, S. 2000, A\&A, 364, 179 (Paper I)

Freire, R., Czarny, J., Felenbok, P., \& Praderie, F. 1977, A\&A, 61, 785

Freire Ferrero, R., Gouttebroze, P., Catalano, S., et al. 1995, ApJ, 439, 1011
Garcia Lopez, R. J., Rebolo, R., Beckman, J. E., \& McKeith, C. D. 1993, A\&A, 273, 482

Gillet, D., Burnage, R., Kohler, D., et al. 1994, A\&AS, 108, 181

Gray, D. 1992, The Observation and Analysis of Stellar Photospheres, 2nd edition (Cambridge University Press), 260

Gunn, A. G., Hall, J. C., Lockwood, G. W., \& Doyle J. G. 1996, A\&A, 305, 146

Hall, J. C., \& Ramsey, L. W. 1992, AJ, 104, 1942

Harper, W. E. 1934, Publ. of Dominion Astr. Obs. vol. VI, 10

Hauschildt, P. H., Allard, F., \& Baron, E. 1999a, ApJ, 512, 377

Hauschildt, P. H., Allard, F., Ferguson, J. W., Baron, E., \& Alexander, D. R. 1999b, ApJ, 525, 871

Heasley, J. N., \& Mihalas, D. 1976, ApJ, 205, 273

Herbig, G. H. 1985, ApJ, 289, 269

Hünsch, M., Schmitt, J. H. M. M., Sterzik, M. F., \& Voges, W. 1999, A\&AS, 135, 319

INES Newsletter, ESA Publication, March 2000 http://ines.vilspa.esa.es

Johnson, H. L., Iriarte, B., Mitchell, R. I., \& Wisniewski, W. Z. 1966, Comm. Lunar Plan. Lab., 4, 99

Lindemann, E., \& Hauck, B. 1973, A\&AS, 11, 119

Micela, G., Sciortino, S., Kashyap, V., Harnden, F. R. Jr., \& Rosner, R. 1996, ApJS, 102, 75

Montes, D., \& Martin, E. L. 1998, A\&AS, 128, 485

Montes, D., Fernández-Figueroa, M. J., De Castro, E., et al. 2000, A\&AS, 146, 103

Moon, T. 1985, Communications from the University of London Obs., 78

Nichols, J. S., \& Linsky, J. L. 1996, AJ, 111, 517

Pavlenko, Y. V., \& Magazzù, A. 1996, A\&A, 311, 961

Prosser, C. F., Randich, S., Stauffer, J. R., Schmitt, J. H. M. M., \& Simon, T. 1996, AJ, 1121570

Radick, R. R., Lockwood, G. W., Skiff, B. A., \& Baliunas, S. L. 1998, ApJS, 118, 239

Reiners, A., Schmitt, J. H. M. M. 2003, A\&A, 398, 647

Roberts, D. H., Lehár, J., \& Dreher, J. W. 1986, AJ, 93, 968

Roman, N. G. 1949, ApJ, 110, 205

Royer, F., Grenier, S., Baylac, M.-O., Gomez, A. E., \& Zorec, J. 2002, A\&A, 393, 897

Rutten, R. G. M., Schrijver, C. J., Lemmens, A. F. P., \& Zwaan, C. 1991, A\&A, 252, 203

Schmitt, J. H. M. M., Golub, L., Harnden, F. R., et al. 1985, ApJ, 290, 307

Schmitt, J. H. M. M., Micela, G., Sciortino, S., et al. 1990, ApJ, 351, 492

Simon, T., \& Landsman, W. 1991, ApJ, 380, 200

Simkin, S. M. 1974, A\&A, 31, 129

Soderblom, D. R., \& Clements, S. D. 1987, AJ, 93, 920

Soderblom, D. R., \& Mayor, M. 1993, AJ, 105, 226

Soderblom, D. R., Pilachowski, C. A., Fedele, S. B., \& Jones, B. F. 1993a, AJ, 105, 2299

Soderblom, D. R., Jones, B. F., Balachandran, S. et al. 1993b, AJ, 106, 1059

Tsikoudi, V., \& Bromage, G. E. 1995, A\&A, 293, 64

Turcotte, S., Richer, J., \& Michaud, G. 1998, ApJ, 504, 559

Vladilo, G., Molaro, P., Crivellari, L., et al. 1987, A\&A, 185, 233

Wolff, S. C., \& Simon, T. 1997, PASP, 109, 759

Zarro, D. M., \& Rodgers, A. W. 1983, ApJS, 53, 815 